\title{
INSIGHT OF AUTISM SCREENING, INTERVENTION AND ITS CORRELATION
}

\author{
A.S. Shminan ${ }^{1}$, S. Sharif ${ }^{2}$, L.J. Choi ${ }^{1}$, N. Fauzan ${ }^{1}$ \\ ${ }^{1}$ Universiti Malaysia Sarawak, Faculty of Cognitive Sciences and Human Development \\ (MALAYSIA) \\ ${ }^{2}$ Universiti Malaysia Sabah Kota Kinabalu, School of Education and Social Development
}

(MALAYSIA)

\begin{abstract}
Autism spectrum disorder is a neurodevelopmental disorder causing an abnormality in one's social, communication and behavior. It is diagnosed based on early-emerging social and communication impairments and the tendency to behave differently than would a normal child. The global statistics reports an increase in the total statistics from year to year. The main question shed light in this article is "What are the existing autism screening tools that have the correlation with autism intervention?". For this objective, a methodology based on a systematic review is done to identify the existing type of autism 'screening' and intervention. The article search is done from early 1990s to the current year and involves peer-reviewed journals. It is important for parents to seek for early assistance if their child shows symptoms of being autistic. The screening process is essential because early detection of autism may lead to early intervention further enabling a child's abilities to be improved. Various screening tools accessible online should be able to tell if a child is autistic. More research should focus on the autism screening process and tools to prove to the public about the reliability of certain available screening tools. The objectives of the research are achieved. A lot of information has been obtained throughout this literature review regarding autism screening and intervention. The instruments used in the screening process are typically inexpensive and simple to administer, score, and interpret. The evaluation of the screening measures is based on sensitivity and specificity. Conclusively, early screening is important because it may contribute to early intervention which will increase the possibility of an autistic child having a better future. There should be more research conducted on autism screening itself and the benefits of having an intervention especially in terms of the social and behavior outcome and family relationship. It is hoped that some information regarding autism can be disseminated to the public and further actions such as screening can be done by parents who are concerned about their child development.
\end{abstract}

Keywords: autism; autism screening tool; autism intervention.

\section{INTRODUCTION}

Autism is a developmental disorder detected based on social and communication impairments that emerge early on, and the tendency to show the behaviour that is different from that of a normal child [1]. The statistics shows that one in 68 U.S. children has an autism spectrum disorder (ASD) [2]. Children as early as 18 months old can already be diagnosed as autistic, so parents need to consult their doctor as soon as possible if their child shows any symptoms of being autistic. Screening test is performed to verify that the child is an autistic child before they move on to the intervention process [3][4]. The child's parents or caregiver can look out for any signs of autism if any symptoms are present or if they are suspicious about the child's behaviour or development. Even though an initial screening can be carried out by accessing screening test or questionnaire that is available online, a potentially autistic child still has to be referred to a family doctor or child psychologist so that full screening can be done. Early intervention for autism is very important to ensure that a child experiences a proper development and to make them stand a chance at leading a normal life [5] . Without early intervention, both parents and the autistic child will tend to feel disappointed as they cannot understand each other's feelings and they will have some trouble communicating. According to Butter et al.[5] shares that there are many types of Autism interventions, treatments and therapies available as each autistic child is distinctive from one another. For example, behavioural intervention is designed to promote appropriate behaviour; development intervention is designed to target the core deficits that each child might have, and psychological intervention includes talking therapy, creative therapy and cognitive behavioural therapy. 